\title{
Discrete Bisector Function and Euclidean Skeleton
}

\author{
Michel Couprie ${ }^{1,2}$ and Rita Zrour ${ }^{1,2}$ \\ 1 Laboratoire A2SI, Groupe ESIEE, BP99, 93162 Noisy-le-Grand Cedex France \\ 2 IGM, Unité Mixte de Recherche CNRS-UMLV-ESIEE UMR 8049 \\ m. couprie@esiee.fr \\ zrour@llaic3.u-clermont1.fr
}

\begin{abstract}
We propose a new definition and an exact algorithm for the discrete bisector function, which is an important tool for analyzing and filtering Euclidean skeletons. We also introduce a new thinning method which produces homotopic discrete Euclidean skeletons. Unlike previouly proposed approaches, this method is still valid in $3 \mathrm{D}$.
\end{abstract}

\section{Introduction}

The notion of skeleton plays a major role in shape analysis. It has been introduced by Blum [5] in 1961 and is the subject of an abundant literature, which deals with both metrical and topological aspects (see e.g. [1, 2, 6, 12, 13, 15, 16, $19,21,25,26]$ ). In this paper, we focus on skeletons in the discrete grid $\mathbb{Z}^{2}$ or $\mathbb{Z}^{3}$, which are centered in the shape with respect to the Euclidean distance, and which have the same topology as the original shape.

Introduced by Talbot and Vincent [25] and generalizing a notion proposed by Meyer 18, the bisector function can play an important role in analyzing and filtering skeletons [1,2,19. Informally, the bisector function associates to each object point $x$ the maximal angle formed by $x$ (as the vertex) and the points of the background which are nearest to $x$. Until now, the algorithms proposed to compute the bisector function in $\mathbb{Z}^{2}$ were based on the use of vectors produced by distance transformation algorithms (e.g., 7]). To each object point, with such algorithms, only one vector indicates the location of a closest background point, and some other points at the same distance may be ignored. In fact, the aim of previous approaches was to compute an approximation of the bisector function as defined in a continuous framework.

This paper contains two original contributions. First, we propose a new definition and an exact algorithm to compute a discrete bisector function. This algorithm was inspired by the methods recently introduced [6, 21] to compute the exact Euclidean medial axis, and is also based on a pre-computed look-up table. Second, we introduce a new thinning method which produces homotopic discrete Euclidean skeletons. Unlike previously proposed approaches, this method is still valid in $3 \mathrm{D}$. 


\section{Basic Notions}

In this section, we recall some basic metrical and topological notions for binary images [8, 15. For simplicity, we limit this presentation to the 2D case.

We denote by $\mathbb{Z}$ the set of integers, by $\mathbb{N}$ the set of nonnegative integers, and by $\mathbb{N}^{*}$ the set of strictly positive integers. We denote by $E$ the discrete plane $\mathbb{Z}^{2}$. A point $x$ in $E$ is defined by $\left(x_{1}, x_{2}\right)$ with $x_{i}$ in $\mathbb{Z}$. Let $x, y \in E$, we denote by $d^{2}(x, y)$ the square of the Euclidean distance between $x$ and $y$, that is, $d^{2}(x, y)=$ $\left(x_{1}-y_{1}\right)^{2}+\left(x_{2}-y_{2}\right)^{2}$. Let $Y \subset E$, we denote by $d^{2}(x, Y)$ the square of the Euclidean distance between $x$ and the set $Y$, that is, $d^{2}(x, Y)=\min \left\{d^{2}(x, y) ; y \in\right.$ $Y$ \}. Let $X \subset E$ (the "object"), we denote by $D_{X}^{2}$ the map from $E$ to $\mathbb{N}$ which associates, to each point $x$ of $E$, the value $D_{X}^{2}(x)=d^{2}(x, \bar{X})$, where $\bar{X}$ denotes the complementary of $X$ (the "background"). The map $D_{X}^{2}$ is called the (squared Euclidean) distance map of $X$. Let $x \in E, r \in \mathbb{N}^{*}$, we denote by $B_{r}(x)$ the ball of (squared) radius $r$ centered on $x$, defined by $B_{r}(x)=\left\{y \in E, d^{2}(x, y)<r\right\}$. Notice that, for any point $x$ in $X$, the value $D_{X}^{2}(x)$ is precisely the radius of a ball centered on $x$ and included in $X$, which is not included in any other ball centered on $x$ and included in $X$.

Let us recall the notion of medial axis (see also [20, 25]). Let $X \subseteq E, x \in X$, $r \in \mathbb{N}^{*}$. A ball $B_{r}(x) \subseteq X$ is maximal for $X$ if it is not strictly included in any other ball included in $X$. The medial axis of $X$, denoted by $\operatorname{MA}(X)$, is the set of the centers of all the maximal balls for $X$ (see Fig. 11, see also Fig. 2).

Efficient algorithms have been proposed to compute exact squared Euclidean distance maps [22,23], and also to extract the exact Euclidean medial axis of a shape, from an exact squared Euclidean distance map and using pre-computed look-up tables [6, 21].

In discrete spaces, it is well known that the topology of the medial axis is generally not the same as the topology of the original object. In particular, if $X$ is connected, $\mathrm{MA}(X)$ is generally not connected (see Fig. 11). Let us now introduce some topological notions in $\mathbb{Z}^{2}$ that we use in the sequel.

We consider the two neighborhood relations $\Gamma_{4}$ and $\Gamma_{8}$ defined by, for each point $x \in E: \Gamma_{4}(x)=\left\{y \in E ;\left|y_{1}-x_{1}\right|+\left|y_{2}-x_{2}\right| \leq 1\right\}, \Gamma_{8}(x)=\left\{y \in E ; \max \left(\mid y_{1}-\right.\right.$ $\left.\left.x_{1}|,| y_{2}-x_{2} \mid\right) \leq 1\right\}$. In the following, we will denote by $n$ a number such that $n=4$ or $n=8$. We define $\Gamma_{n}^{*}(x)=\Gamma_{n}(x) \backslash\{x\}$. The point $y \in E$ is $n$-adjacent to $x \in E$ if $y \in \Gamma_{n}^{*}(x)$. An $n$-path is a sequence of points $x_{0} \ldots x_{k}$ with $x_{i} n$-adjacent to $x_{i-1}$ for $i=1 \ldots k$.

Let $X$ be a non-empty subset of $E$. We say that two points $x, y$ of $X$ are $n$-connected in $X$ if there is an $n$-path in $X$ between these two points. This defines an equivalence relation. The equivalence classes for this relation are the $n$-connected components of $X$, or $n$-components in short. The set $X$ is said to be $n$-connected if it consists of exactly one $n$-component. The set composed of all $n$-components of $X$ which are $n$-adjacent to a point $x$ is denoted by $C_{n}[x, X]$.

In order to have a correspondence between the topology of $X$ and the topology of $\bar{X}$, we have to consider two different kinds of adjacency for $X$ and $\bar{X}$ [15]: if we use the $n$-adjacency for $X$, we must use the $\bar{n}$-adjacency for $\bar{X}$, with $(n, \bar{n})=$ $(8,4)$ or $(4,8)$. In the sequel, we assume that the adjacency pair $(n, \bar{n})=(8,4)$ 


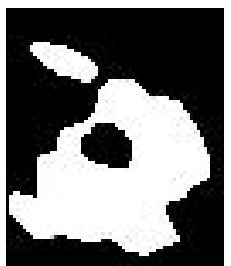

(a)

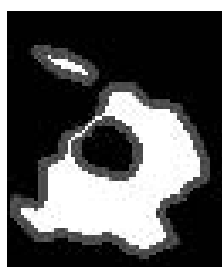

(b)

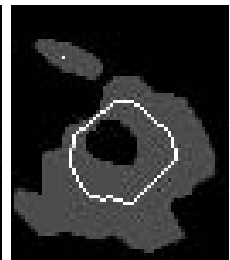

(c)

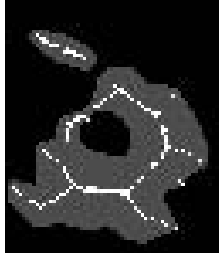

(d)

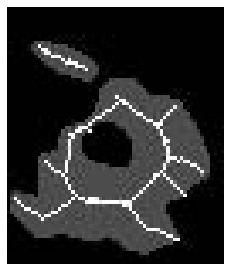

(e)

Fig. 1. (a): a set $X$ (in white); (b): an homotopic thinning of $X$; (c): ultimate homotopic skeleton of $X ;(\mathrm{d})$ : medial axis of $X$; (e): ultimate homotopic skeleton of $X$ constrained by the medial axis of $X$. In $(\mathrm{b}, \mathrm{c}, \mathrm{d}, \mathrm{e})$ the original set $X$ appears in dark gray for comparison

has been chosen and we do not write the subscripts $n, \bar{n}$ unless necessary; but the results also hold for $(n, \bar{n})=(4,8)$.

Informally, a simple point $p$ of a discrete object $X$ is a point which is "inessential" to the topology of $X$. In other words, we can remove the point $p$ from $X$ without "changing the topology of $X$ ". The notion of simple point is fundamental to the definition of topology-preserving transformations in discrete spaces. We now give a definition and a local characterization of simple points in $E=\mathbb{Z}^{2}$. For the 3D case, see 3 .

The point $x \in X$ is simple (for $X$ ) if each $n$-component of $X$ contains exactly one $n$-component of $X \backslash\{x\}$ and if each $\bar{n}$-component of $\bar{X} \cup\{x\}$ contains exactly one $\bar{n}$-component of $\bar{X}$. Let $X \subseteq E$ and $x \in E$, the two connectivity numbers are defined as follows (\#X stands for the cardinality of $X$ ):

$$
T(x, X)=\# C_{n}\left[x, \Gamma_{8}^{*}(x) \cap X\right] ; \bar{T}(x, X)=\# C_{\bar{n}}\left[x, \Gamma_{8}^{*}(x) \cap \bar{X}\right] .
$$

The following property allows us to locally characterize simple points [15, 3], hence to implement efficiently topology preserving operators:

$$
x \in E \text { is simple for } X \subseteq E \Leftrightarrow T(x, X)=1 \text { and } \bar{T}(x, X)=1 .
$$

Let $X$ be any finite subset of $E$. The subset $Y$ of $E$ is an homotopic thinning of $X$ if $Y=X$ or if $Y$ may be obtained from $X$ by iterative deletion of simple points. We say that $Y$ is an ultimate homotopic skeleton of $X$ if $Y$ is an homotopic thinning of $X$ and if there is no simple point for $Y$.

Let $C$ be a subset of $X$. We say that $Y$ is an ultimate homotopic skeleton of $X$ constrained by $C$ if $C \subseteq Y$, if $Y$ is an homotopic thinning of $X$ and if there is no simple point for $Y$ in $Y \backslash C$ (see e.g. [12,26]). The set $C$ is called the constraint set relative to this skeleton.

\section{The Bisector Function: New Definition and Exact Algorithm}

Let $X$ be a non-empty subset of $E$, and let $x \in X$. The downstream of $x$ in $X$, denoted by $\operatorname{Ds}(x, X)$ or by $\operatorname{Ds}(x)$ when no confusion may occur, is the set of points $y$ of $\bar{X}$ which are at minimal distance from $x ;$ more precisely, $\operatorname{Ds}(x, X)=$ $\left\{y \in \bar{X}, \forall z \in \bar{X}, d^{2}(y, x) \leq d^{2}(z, x)\right\}$. For example in Fig. 2, we have $\operatorname{Ds}(x)=$ 


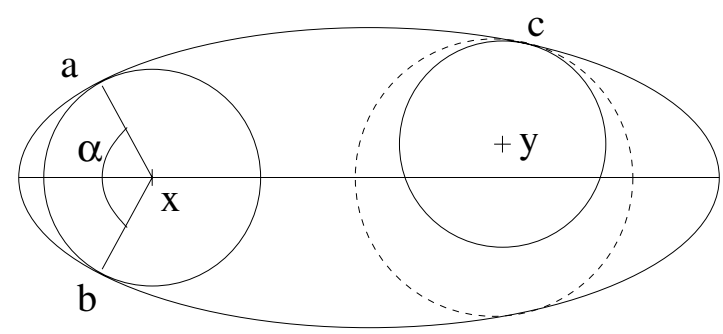

Fig. 2. A set $X$ (full ellipsis, represented by its border) and its medial axis (horizontal line), a point $x$ and its downstream $\{a, b\}$, a point $y$ and its downstream $\{c\}$. Notice that $y$ does not belong to the medial axis, since no ball centered on $y$ and included in $X$ is maximal for $X$

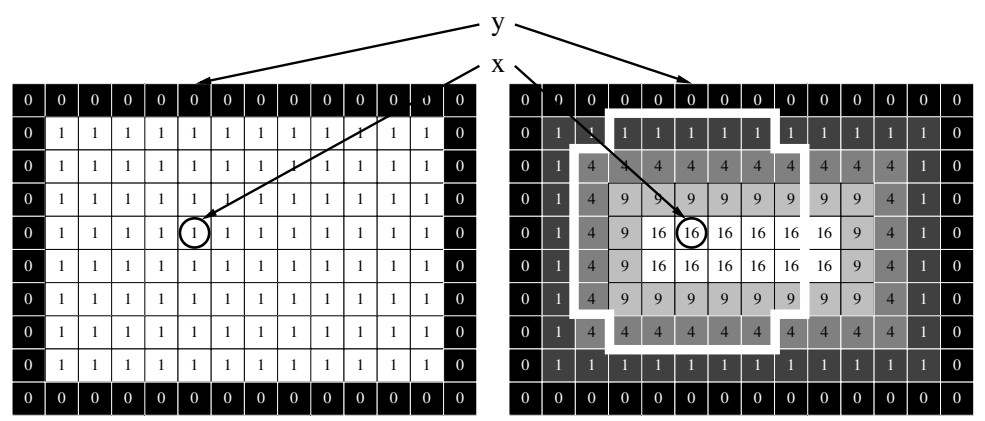

Fig. 3. A set $X$ and its distance map $D_{X}^{2}$. The point $x$ belongs to the medial axis of $X$ (it is the center of a maximal ball for $X$, delineated in white). The downstream of $x$ is the singleton $\{y\}$

$\{a, b\}$ and $\operatorname{Ds}(y)=\{c\}$. The bisector angle of a point $x$ in $X$ can be defined informally, in the continuous framework, as the maximal unsigned angle formed by $x$ (as the vertex) and any two points in $\operatorname{Ds}(x)$. In Fig. 2, the bisector angle of $x$ is $\alpha$, and the bisector angle of $y$ is 0 .

In the continuous framework, a point $x$ which belongs to the medial axis is characterized by the fact that $\operatorname{Ds}(x)$ contains strictly more than one point, in other words, its bisector angle is non-zero. In the discrete case such a characterization is not possible, due to configurations like Fig. 3, which are very common. At least, we want such medial axis points to have a non-zero bisector angle; this motivates the introduction of the following notion.

Let $X \subset E$, and let $x \in X$. The extended downstream of $x$ in $X$, denoted by $\operatorname{EDs}(x, X)$ or by $\operatorname{EDs}(x)$ when no confusion may occur, is the union of the sets $\operatorname{Ds}(y, X)$, for all $y$ in $\Gamma_{4}(x)$.

Now we can propose our definition of the discrete bisector function. 
Let $X \subset E$, and let $x \in X$. The bisector angle of $x$ in $X$, denoted by $\theta_{X}(x)$, is the maximal unsigned angle between the vectors $\overrightarrow{x y}, \overrightarrow{x z}$, for all $y, z$ in $\operatorname{EDs}(x)$. The bisector function of $X$, denoted by $\theta_{X}$, is the function which associates to each point $x$ of $X$, its bisector angle in $X$.

We now propose a method to exactly and efficiently compute the bisector function, with the help of a squared Euclidean distance map. The following property, which may be easily established, is the key of the method.

Property 1. Let $X \subset E$, let $x \in X$, and let $y \in E$. The point $y$ belongs to the downstream of $x$ if and only if $d^{2}(x, \bar{X})=d^{2}(x, y)$ and $d^{2}(y, \bar{X})=0$.

Now, observe that for any given point $x \in X$, the value $R=d^{2}(x, \bar{X})=$ $D_{X}^{2}(x)$ can be read in a pre-computed squared Euclidean distance map. The positions of the points $y$ such that $R=d^{2}(x, y)$ can be found by solving the diophantine equation $\left(x_{1}-y_{1}\right)^{2}+\left(x_{2}-y_{2}\right)^{2}=R$, or more simply $\alpha^{2}+\beta^{2}=R$. This amounts to compute the different decompositions of a given integer into a sum of two squares (or three squares in 3D), a problem which has been studied extensively (see [14).

Furthermore, these decompositions can also be pre-computed and stored in a look-up table. The program which computes this look-up table is very simple (see Annex). Notice that the same look-up table was used by Fontoura-Costa et al. to compute an exact Euclidean dilation[17]. Once all decompositions are known, one must take into account the different symmetries of the space (their number is 8 for $\mathbb{Z}^{2}, 48$ for $\mathbb{Z}^{3}$ ) and check those positions $y$ which satisfy the second condition of prop. 1. $d^{2}(y, \bar{X})=0$, again using the distance map.

The following algorithm summarizes the computation of the extended downstream of a given point $x \in X$.

\section{Procedure ExtendedDownstream (Input $D_{X}^{2}, x$, Output $E D s$ )} 01. EDs $\leftarrow \emptyset$

02. ForEach $v \in \Gamma_{4}(x)$

03. $T \leftarrow\left\{\left(z_{1}, z_{2}\right) \in \mathbb{Z}^{2} ; z_{1} \geq z_{2} \geq 0 ; z_{1}^{2}+z_{2}^{2}=D_{X}^{2}(v)\right\}$ (from LUT)

04. $T^{\prime} \leftarrow\left\{\left(z_{1}, z_{2}\right) \in \mathbb{Z}^{2} ;\left(\left|z_{1}\right|,\left|z_{2}\right|\right)\right.$ is a permutation of an element of $\left.T\right\}$

05. ForEach $z \in T^{\prime}$ Do

06. If $D_{X}^{2}(v+z)=0$ Then $E D s \leftarrow E D s \cup\{v+z\}$

The last step to obtain the bisector angle consists in the computation of the maximum unsigned angle between all the pairs of vectors $\{\overrightarrow{x y}, \overrightarrow{x z}\}$ for all $y, z$ in $\operatorname{EDs}(x)$. If we denote by $k$ the number of points in $\operatorname{EDs}(x)$, the number of such pairs is quadratic with respect to $k$, more precisely, it is equal to $k(k-1) / 2$. By normalizing all these vectors, we can easily see that the problem of finding a maximum angle reduces to the problem of finding a maximum diameter of a convex polygon in 2D. This last problem has been solved in 1978 by Shamos 24, who provided a simple linear-time algorithm (that is, in $O(k)$ ). In 3D, the problem is more complicated but some efficient algorithms (in $O(n \log n)$ or less) have been proposed for the maximal diameter of a set of points, see e.g. 4. However, in practice, the mean cardinal of the extended downstream for a given shape is 

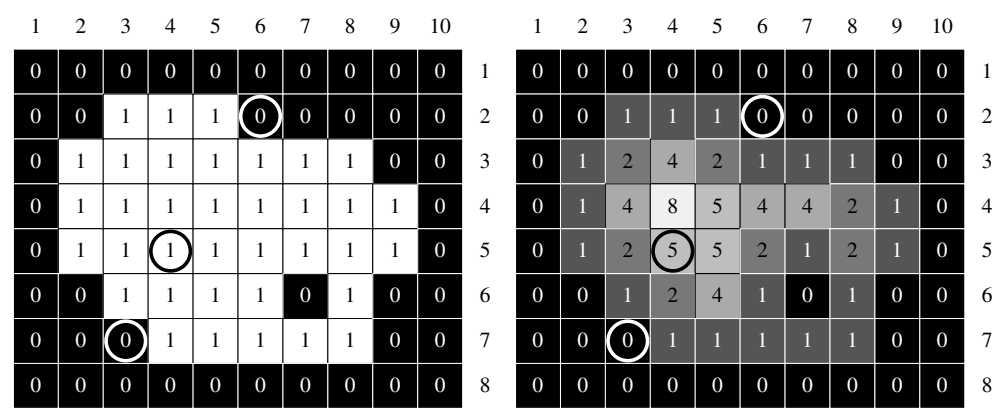

Fig. 4. Left: a set $X$ (in white); Right: the squared Euclidean map of $X$. The point $x=(4,5)$ is circled in black, the points $y_{1}=(6,2)$ and $y_{2}=(3,7)$ are circled in white

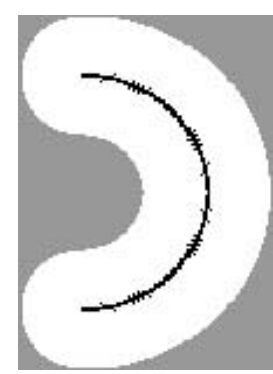

(a)

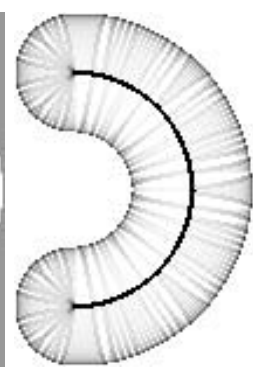

(b)

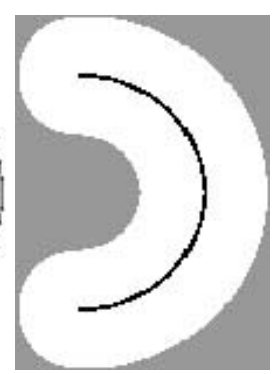

(c)

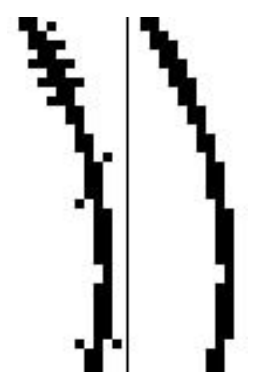

(d)

Fig. 5. (a): a set $X$ and its medial axis (in black); (b): the bisector function $\theta_{X}$ (dark colors correspond to wide angles); (c): filtered medial axis, based on the values of $\theta_{X}$; (d): detail of the non-filtered and filtered medial axis

usually quite small. Thus, the straightforward algorithm which considers all the pairs of points in the extended downstream is the best choice in most cases.

We illustrate this procedure using the example of Fig. 4. We consider the point $x=(4,5)$, which is circled in black in the figure. Thus, $\Gamma_{4}(x)=\left\{x, x_{1}\right.$, $\left.x_{2}, x_{3}, x_{4}\right\}$ with $x_{1}=(5,5), x_{2}=(4,4) x_{3}=(3,5), x_{4}=(4,6)$. Let us begin with $x=(4,5)$, and $D_{X}^{2}(x)=5$. From the Look-up table (see Fig. 9), we see that the only decomposition of 5 into a sum of two squares is $2^{2}+1^{2}$. Applying the 8 symmetries of $\mathbb{Z}^{2}$, we find the offsets $\{(2,1),(-2,1),(-2,-1),(2,-1)$, $(1,2),(-1,2),(-1,-2),(1,-2)\}$, hence the points $\{(6,6),(2,6),(2,4),(6,4)$, $(5,7),(3,7),(3,3),(5,3)\}$. Among these points, only $(2,6)$ and $(3,7)$ are in the downstream of $x$ since $D_{X}^{2}[(2,6)]=D_{X}^{2}[(3,7)]=0$. Carrying on with the points $x_{1}, x_{2}, x_{3}, x_{4}$, we find that $\operatorname{EDs}(x)=\{(2,6),(3,7),(7,6),(2,2),(6,2)\}$. Let $y_{1}=(6,2)$ and $y_{2}=(3,7)$, it may be easily checked that the maximum unsigned angle corresponds to the couple of vectors $\left\{x \vec{y}_{1}, x \vec{y}_{2}\right\}$ and is close to $3.02 \mathrm{rad}$.

In Fig. [5] we show a set $X$ together with its medial axis (a) and the bisector function $\theta_{X}(\mathrm{~b})$. We illustrate the use of this function to eliminate spurious points of the medial axis: in (c), we show the points of the medial axis (in black) 


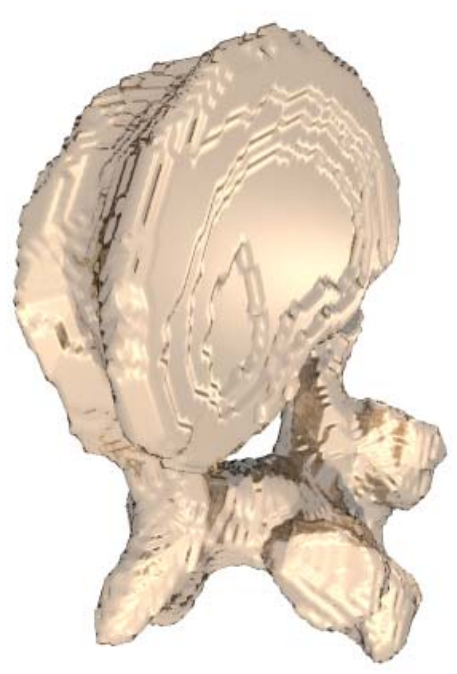

(a)

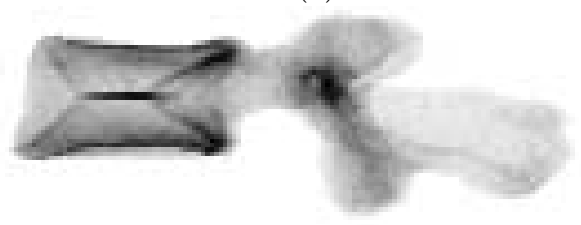

(c)

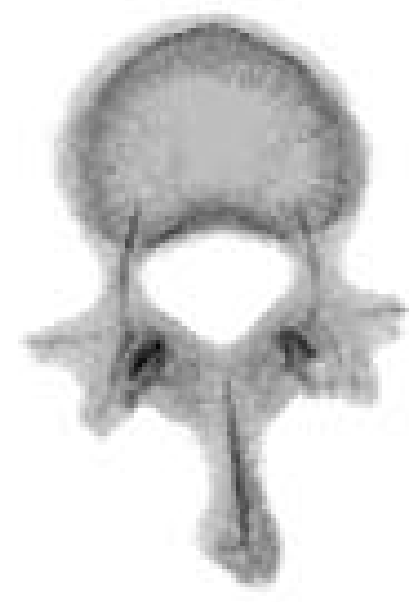

(b)

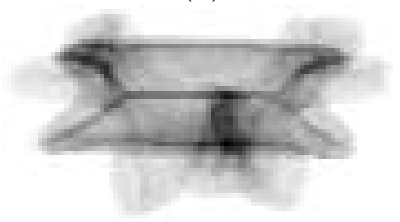

(d)

Fig. 6. (a): a view of a subset $X$ of $\mathbb{Z}^{3}$ (vertebra), generated thanks to a topologically sound "Marching Cubes-like" algorithm 11]; (b,c,d): the bisector function, illustrated in an "X-ray" manner: the gray level of a point corresponds to the average of the bisector angles on a straight line parallel to one of the three axes

which have a bisector angle greater than 0.7 rad. A zoomed detail of both axes is shown in (d). Notice that only the bisector angles of the medial axis points need to be computed for this application.

The definition and exact computation of this discrete bisector function can be straightforwardly extended to $\mathbb{Z}^{3}$. To conclude this section, we present in Fig. 6] an illustration of our bisector function of a three-dimensional object (a vertebra). As a matter of fact, the number of decompositions of an integer $k$ grows is a "reasonable way" when $k$ increases, see [10] for more details. The size of the original image of Fig. [6] is $122 \times 144 \times 53=931104$ points, among which 103302 are object points. On a standard PC, computing the bisector function on all object points for this image takes $2.9 \mathrm{~s}$, and $0.5 \mathrm{~s}$ for only medial axis points. The look-up table used for processing this image occupies 82750 bytes of memory. 


\section{New Distance-Guided Homotopic Thinning Algorithm}

The skeletonization methods which are based on homotopic thinnings, in the sense of section 2, provide a formal guarantee that the skeleton and the original object have the same topology. The simplest such method consists in computing an ultimate homotopic skeleton of the object $X$ constrained by the medial axis of $X$, that is, removing iteratively simple points from $X$ which do not belong to $\mathrm{MA}(X)$, taking the distance map as a priority function in order to select first the points which are closest to the background. This can be done using the following procedure, with $P=D_{X}$ and $Y=\operatorname{MA}(X)$.

Procedure UltimateSkeleton (Input $X, P, Y$, Output $Z$ )

01. $Z \leftarrow X$

02. $Q \leftarrow\{(P(x), x)$; where $x$ is any point of $X \backslash Y\}$

03. While $Q \neq \emptyset$ Do

04. choose $(p, x)$ in $Q$ such that $p$ is minimal

05. If $x$ is simple for $Z$ Then

06. $Z \leftarrow Z \backslash\{x\}$

07. $Q \leftarrow Q \cup\{(P(y), y)$; where $y \in \Gamma(x) \cap(Z \backslash Y)\}$

The drawback of this method has been well analyzed in [25]. Roughly speaking the method does not guarantee that points of the skeleton outside the medial axis are "well centered" in the object; more precisely, such a point may have a null or quasi-null bisector angle.

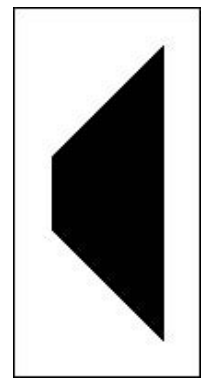

(a)

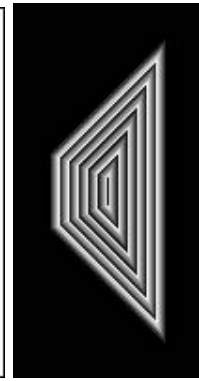

(b)

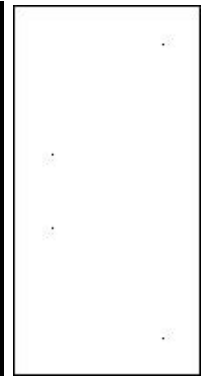

(c)

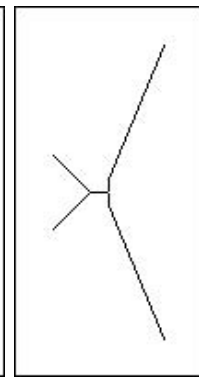

(d)

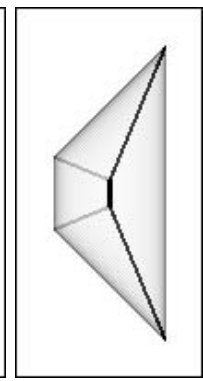

(e)

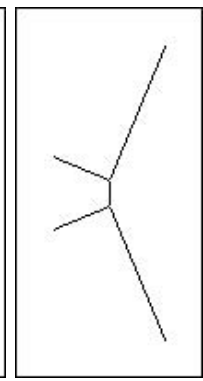

(f)

Fig. 7. (a): a set $X$ (in black); (b): isolines of the Euclidean map $D_{X}$; (c): a constraint set $Y$ (four points located at the "corners" of the shape); (d): homotopic skeleton of $X$ guided by $D_{X}^{2}$ and constrained by $Y$; (e): the bisector function of $X$; (f): the result of our algorithm EuclideanSkeleton

Let us consider the object $X$ in Fig. 7 a, and the constraint set $Y$ in Fig. 7 f, which idealizes a filtered medial axis of $X$. The result of UltimateSkeleton $(X$, $\left.D_{X}, Y\right)$ is depicted in Fig. [7d. The isolines of the distance map $D_{X}$ and the bisector function of $X$ are depicted in Fig. $7 \mathrm{~b}$,e respectively, for comparison. 


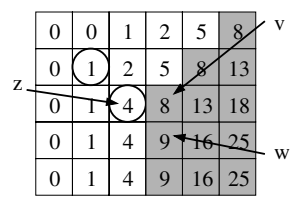

Fig. 8. A detail of the preceding example

To understand what happens, let us concentrate on a detail of the above example, depicted in Fig. 8. The numbers correspond to the distance map values. The circled point with value 1 is one of the points belonging to the constraint set $Y$. Suppose that all the points with a value below 8 have been processed by the homotopic thinning algorithm. At this step, the points in gray are still in $X$, as well as the two circled points (the point at 1 because it belongs to $Y$, and the one at 4 because it is not a simple point). All other points are not in $X$. Obviously, the point $v$ at 8 adjacent to $z$ at 4 will be selected before its neighbor $w$ at 9 , and since it will be a simple point at this stage, it will be deleted. Such a behaviour will be reproduced at later stages, generating a diagonal branch of the skeleton, and is in contradiction with a property of the skeleton in the continuous framework: informally, skeleton branches follow lines of steepest slope of the Euclidean distance map. Let us compute the slopes of the segments $z v$ and $z w$ in our example: $(\sqrt{8}-\sqrt{4}) / 1 \approx 0.83$, and $(\sqrt{9}-\sqrt{4}) / \sqrt{2} \approx 0.71$. Thus, the point $v$ should be kept in the skeleton and the point at $w$ deleted according to this criterion.

In [25], Talbot and Vincent propose the following strategy to cope with this problem. During the thinning process guided by the distance map, having detected a point $x$ which belongs to the skeleton (either a point of the constraint set or a non-simple point), the neighbor of $x$ which corresponds to the steepest ascending slope is dynamically added to the constraint set. Although this method gives satisfactory results in $2 \mathrm{D}$, it cannot be extended to the $3 \mathrm{D}$ case.

We propose another strategy which gives equivalent results in $2 \mathrm{D}$ and which also applies to the $3 \mathrm{D}$ case. The idea is to define a priority function which takes into account both the distance map and an auxiliary function defined in the neighborhood of each dynamically detected skeleton point. Let $x$ be such a point, then to any neighbor $y$ of $x$ which is still in $X$ and not in the constraint set $Y$, we associate the value $p_{y}=D_{X}(x)+\left(D_{X}(y)-D_{X}(x)\right) / d(x, y)$, with $D_{X}(x)=\sqrt{D_{X}^{2}(x)}$ and $d(x, y)=\sqrt{d^{2}(x, y)}$. The new priority function, for the point $y$, is defined as $\min \left(p_{y}, D_{X}(y)\right)$. We see that $\left(D_{X}(y)-D_{X}(x)\right) / d(x, y)$ is the slope of $x y$, thus the neighbors of $x$ will be examined in increasing order of slope, since the value $p_{y}$ is always less or equal to the corresponding distance value $D_{X}(y)$ (for all $x, y$ in $\mathbb{Z}^{2}$ or $\mathbb{Z}^{3}$ with $x \neq y$, we have $d(x, y) \geq 1$ ).

For example, in the above situation, we have $D_{X}(v)=\sqrt{8} \approx 2.83, D_{X}(w)=$ $3, p_{v}=\sqrt{4}+(\sqrt{8}-\sqrt{4}) / 1=\sqrt{8}$ and $p_{w}=\sqrt{4}+(\sqrt{9}-\sqrt{4}) / \sqrt{2} \approx 2.71$; thus the point $w$ will be selected before $v$ with this strategy. Our algorithm is given below. 
Procedure EuclideanSkeleton (Input $X, D_{X}, Y$, Output $Z$ )

01. $Z \leftarrow X$

02. $Q \leftarrow\left\{\left(D_{X}(x), x\right)\right.$ where $x$ is any point of $\left.Z \backslash Y\right\}$

03. $R \leftarrow\left\{\left(p_{x}, x\right)\right.$ where $x$ is any point of $Z \backslash Y$ adjacent to $Y$,

04. $\quad$ and where $\left.p_{x}=\min \left\{D_{X}(z)+\left(D_{X}(x)-D_{X}(z)\right) / d(x, z), z \in Y\right\}\right\}$

05. While $Q \neq \emptyset$ Or $R \neq \emptyset$ Do

06. choose $(p, x)$ in $Q \cup R$ such that $p$ is minimal

07. If $x \in Z \backslash Y$ Then

08. If $x$ is simple for $Z$ Then

09. $\quad Z \leftarrow Z \backslash\{x\}$

10. Else

11. $Y \leftarrow Y \cup\{x\}$

12. $\quad R \leftarrow R \cup\left\{\left(p_{y}, y\right)\right.$, where $y \in \Gamma(x) \cap(Z \backslash Y)$

13. $\quad$ and where $\left.p_{y}=D_{X}(x)+\left(D_{X}(y)-D_{X}(x)\right) / d(x, y)\right\}$

In Fig. 17, we see the result of this algorithm applied to the preceding example. Compare the shape of this skeleton with the distance map and with the bisector function of $X$ depicted in $7 \mathrm{~b}$,e respectively. The complexity of this algorithm depends on the data structure used to represent the sets $Q$ and $R$. To be more precise, this data structure must allow to perform the choice in line 6 efficiently, and also the insertions in lines 11 and 12 . Using for example balanced binary trees [9], the overall complexity of the algorithm is in $O(n \log n)$, where $n$ is the number of image points.

\section{Conclusion}

We introduced a new definition and an exact algorithm for the discrete bisector function, and proposed a new thinning algorithm which produces homotopic discrete Euclidean skeletons. Both constitute significant improvements with respect to previous approaches, and apply to the $2 \mathrm{D}$ and $3 \mathrm{D}$ cases.

In an extended version of this paper [10, we will analyse more deeply the difference between the proposed approach and the previous ones, we will present more results and applications (especially with "real world" data and in 3D), and we will analyze the performance of these algorithms both in terms of time and memory space.

\section{References}

1. D. Attali, J.O. Lachaud: "Delaunay Conforming Iso-surface, Skeleton Extraction and Noise Removal", Computational Geometry: Theory and Applications, Vol. 19, pp. 175-189, 2001.

2. D. Attali, A. Montanvert: "Modelling noise for a better simplification of skeletons", Procs. International Conference on Image Processing, Vol. 3, pp. 13-16, 1996.

3. G. Bertrand: "Simple points, topological numbers and geodesic neighborhoods in cubic grids", Pattern Recognition Letters, Vol. 15, pp. 1003-1011, 1994. 
4. S.N. Bespamyatnikh, "An efficient algorithm for the three-dimensional diameter problem", Procs. ACM-SIAM symp. on discrete algorithms, pp. 137-146, 1998.

5. H. Blum, "An associative machine for dealing with the visual field and some of its biological implications", Biological prototypes and synthetic systems, Vol. 1, pp. 244-260, 1961.

6. G. Borgefors, I. Ragnemalm, G. Sanniti di Baja, "The Euclidean distance transform: finding the local maxima and reconstructing the shape", Procs. of the 7th Scand. Conf. on image analysis, Vol. 2, pp. 974-981, 1991.

7. P.E. Danielsson: "Euclidean distance mapping", Computer Graphics and Image Processing, 14, pp. 227-248, 1980.

8. J.M. Chassery, A. Montanvert: Géométrie discrète, Hermès, 1991.

9. T.H. Cormen, C.E. Leiserson, R.L. Rivest: Introduction to algorithms, MIT Press, 1990.

10. M. Couprie, R. Zrour: "Discrete bisector function and Euclidean skeleton in 2D and 3D", report IGM2004-12 of the Institut Gaspard Monge (University of Marnela-Vallée), http://www-igm.univ-mlv.fr/LabInfo/rapportsInternes/2004/12.pdf, 2004.

11. X. Daragon, M. Couprie, G. Bertrand: "Discrete frontiers", Springer (Ed.), Discrete Geometry for Computer Imagery, Springer LNCS, Vol. 2886, pp. 236-245, 2003.

12. E.R. Davies, A.P.N. Plummer: "Thinning algorithms: a critique and a new methodology", Pattern Recognition, Vol. 14, pp. 53-63, 1981.

13. Y. Ge, J.M. Fitzpatrick: "On the generation of skeletons from discrete Euclidean distance maps", IEEE Trans. on Pattern Analysis and Machine Intelligence, Vol. 18, No. 11, pp. 1055-1066, 1996.

14. G.H. Hardy, E.M. Wright: An introduction to the theory of numbers, Oxford University Press, 1938.

15. T. Yung Kong, A. Rosenfeld: "Digital topology: introduction and survey", Computer Vision, Graphics and Image Processing, Vol. 48, pp. 357-393, 1989.

16. L. Lam, S-W. Lee, C.Y. Suen: "Thinning methodologies - a comprehensive survey", IEEE PAMI, Vol. 14, No. 9, pp. 869-885, 1992.

17. M. Luppe, L. da Fontoura Costa, V. Obac Roda: "Parallel implementation of exact dilations and multi-scale skeletonization", Real-Time Imaging, Vol. 9, pp. 163-169, 2003.

18. F. Meyer, Cytologie quantitative et morphologie mathématique, $\mathrm{PhD}$ thesis, École des mines de Paris, 1979.

19. G. Malandain, S. Fernández-Vidal, "Euclidean Skeletons", Image and vision computing, Vol. 16, pp. 317-327, 1998.

20. A. Rosenfeld, A.C. Kak: Digital Image processing, Academic Press, 1982.

21. E. Rémy, E. Thiel: "Exact Medial Axis with Euclidean Distance", to appear in Image and Vision Computing, 2004.

22. T. Saito, J.I. Toriwaki: "New algorithms for Euclidean distance transformation of an $n$-dimensional digitized picture with applications", Pattern Recognition, Vol. 27, pp. 1551-1565, 1994.

23. T. Hirata: "A unified linear-time algorithm for computing distance maps", Information Processing Letters, Vol. 58(3), pp. 129-133, 1996.

24. M.I. Shamos: Computational geometry, PhD thesis, Yale University, 1978.

25. H. Talbot, L. Vincent: "Euclidean skeletons and conditional bisectors", Proceedings of VCIP'92, SPIE, Vol. 1818, pp. 862-876, 1992.

26. L. Vincent: "Efficient Computation of Various Types of Skeletons", Proceedings of Medical Imaging V, SPIE, Vol. 1445, pp. 297-311, 1991. 


\section{Annex: Building the Look-Up Table}

Procedure Build2dLUT (Input $N$, Output LUT)

01. $n \leftarrow\lceil\sqrt{N}\rceil$; For $i$ From 0 To $N$ Do LUT $[\mathrm{i}] \leftarrow \emptyset$

02. For $x$ From 0 To $n$ Do

02. For $y$ From 0 To $x$ Do

03.

$$
i \leftarrow x^{2}+y^{2}
$$

04.

If $i \leq N$ Then

05.

$$
\operatorname{LUT}[\mathrm{i}] \leftarrow \operatorname{LUT}[\mathrm{i}] \cup\{(x, y)\}
$$

$\begin{array}{lllll}0:(0,0) & 8:(2,2) & 17:(4,1) & 29:(5,2) & 40:(6,2) \\ 1:(1,0) & 9:(3,0) & 18:(3,3) & 32:(4,4) & 41:(5,4) \\ 2:(1,1) & 10:(3,1) & 20:(4,2) & 34:(5,3) & 45:(6,3) \\ 4:(2,0) & 13:(3,2) & 25:(4,3),(5,0) & 36:(6,0) & 49:(7,0) \\ 5:(2,1) & 16:(4,0) & 26:(5,1) & 37:(6,1) & 50:(5,5),(7,1)\end{array}$

Fig. 9. Look-up table (2D case): numbers 0 to 50

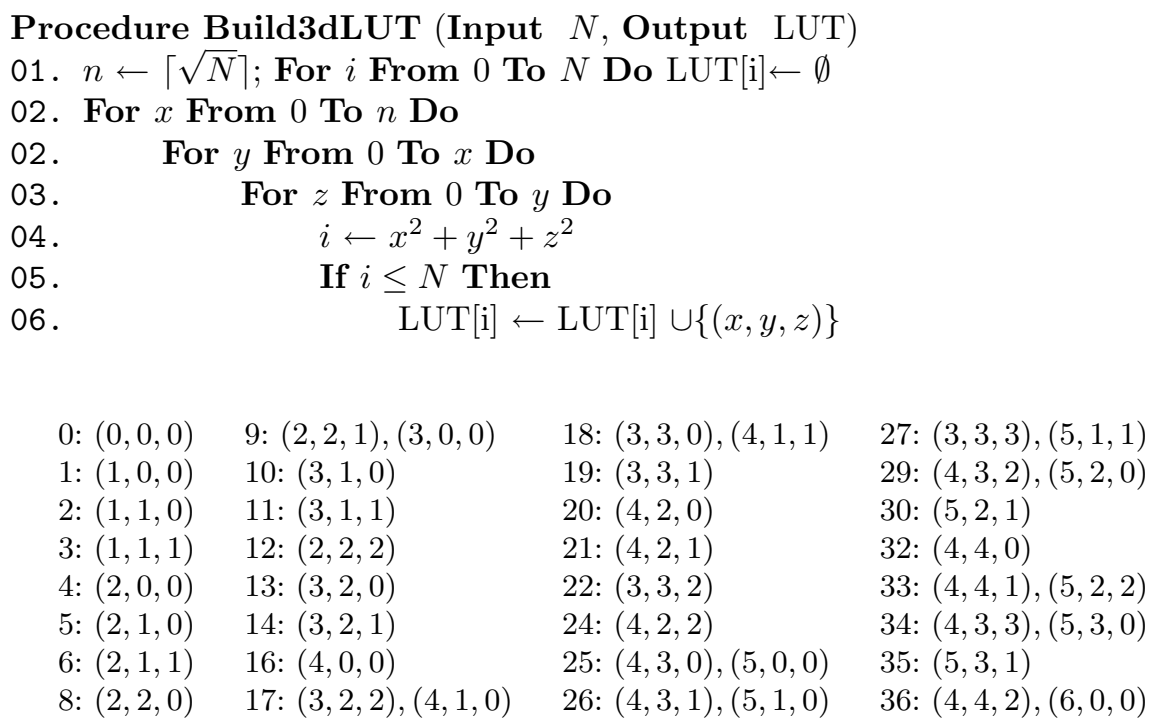

Fig. 10. Look-up table (3D case): numbers 0 to 36 